\title{
SINTOMAS VOCAIS E PERFIL DE PROFESSORES EM UM PROGRAMA DE SAÚDE VOCAL
}

\section{Vocal symptoms and profile of teachers in a vocal health program}

\author{
Karin Choi-Cardim (1), Mara Behlau (2), Fabiana Zambon ${ }^{(3)}$
}

\section{RESUMO}

Objetivo: analisar sintomas vocais de dois grupos de professores que foram avaliados em momentos distintos de um programa de saúde vocal. Métodos: correlacionar condições de trabalho e hábitos com o número de sintomas vocais apresentados por 411 professores, agrupados em G1 (256 sujeitos a serem submetidos ao programa preventivo) e G2 (155 sujeitos a serem submetidos ao programa preventivo e de tratamento). Resultados: em ambos os grupos observou-se predomínio de mulheres $(p=0,550)$, entre 31 e 40 anos $(p=0,557)$, lecionando para mais de um grau de ensino $(p=0,345)$ com até 30 alunos/sala $(p=0,521)$, com presença de ruído no trabalho $(p=0,660)$, que relataram cuidados vocais $(p=0,231)$ e utilizavam voz extra-profissionalmente $(p=0,713)$, não tabagistas $(p=$ $0,010)$ nem alcoolistas $(p=0,029)$. Em contrapartida, no $G 1$ observou-se carga horária diária de trabalho de até 5 horas, enquanto a maior parte do G2 trabalhava de 6 a 10 horas $(p<0,001)$. Enquanto $51 \%$ do G1 não procuraram otorrinolaringologista/ fonoaudiólogo por alterações vocais, $68,38 \%$ do G2 já o haviam feito $(p<0,001)$. O número de sintomas vocais foi elevado para ambos $(\geq 4)$ os grupos; sendo a média de 3,5 (57\%) para G1 e 5,8 (98,05\%) para G2 - ( $<<0,001)$. Conclusão: apesar de perfis semelhantes, houve média maior de sintomas vocais no G2, demonstrando que estes procuraram o serviço fonoaudiológico com maior risco de alteração vocal, possivelmente devido à mudança de intervenção do programa que, oferecendo a reabilitação vocal, atraiu professores com mais alterações. Assim, são importantes programas de saúde vocal tanto focados em prevenção quanto tratamento vocal, pois contribuirão para a qualidade de trabalho e de vida desses profissionais.

DESCRITORES: Voz; Sintomas; Hábitos; Condições de Trabalho; Distúrbios da Voz

\section{INTRODUÇÃO}

Os professores usam a voz como instrumento de trabalho ${ }^{1-3}$ e constituem um dos grupos de maior risco para o desenvolvimento de uma alteração vocal ${ }^{4}$. Essas alterações vocais muitas vezes agravam-se com o uso abusivo ou mau uso da voz, problemas respiratórios ou tabagismo ${ }^{5}$.

(1) Fonoaudióloga; Consultora em Comunicação, São Paulo, SP; Especialista em voz pelo Conselho Federal de Fonoaudiologia.

(2) Fonoaudióloga; Professora do Curso de Especialização em voz do Centro de Estudos da Voz, CECEV, São Paulo, SP; Doutora em Distúrbios da Comunicação Humana pela Universidade Federal de São Paulo.

(3) Fonoaudióloga; Coordenadora do Programa de Saúde Vocal do Sindicato dos Professores de São Paulo, SINPRO, SP; Professora do Curso de Especialização em voz do Centro de Estudos da Voz, CECEV, São Paulo, SP; Especialista em voz.

Conflito de interesses: inexistente
Muitos destes profissionais lecionam para um grande número de alunos por sala de aula, em condições inadequadas ou ambiente ruidoso, enfrentando uma extensa carga horária diária de trabalho ${ }^{6}$, sendo possível que estes fatores contribuam para uma maior incidência de sintomas e alterações vocais ${ }^{4}$.

As queixas relacionadas à voz mais comuns em professores são: fadiga vocal, perda da voz, dor em região de garganta e rouquidão. Em muitos casos, o professor não tem acesso à informação e prevenção sobre a saúde vocal, o que contribui para que a prevalência desses sintomas seja alta nessa categoria profissional ${ }^{5,6}$.

Considerando a necessidade de promoção da saúde vocal e visando diminuir a incidência de sintomas e disfonia entre os professores, em 2001 o serviço fonoaudiológico de uma instituição de atendimento a professores da rede particular de ensino 
iniciou o Programa de Saúde Vocal - PSV para o acompanhamento destes profissionais ${ }^{4}$.

A partir de 2004 este programa passou a oferecer, além da abordagem preventiva (orientações e avaliações vocais), aprimoramento e tratamento vocal. Com a mudança no atendimento do PSV houve a necessidade de uma análise dos professores que procuravam o programa, para adequá-lo às necessidades dessa população.

Portanto, o presente trabalho se propõe a comparar o perfil de professores que procuraram o PSV em duas etapas: quando o programa só oferecia avaliação e orientação vocal (2001-2003) e quando o programa passou a oferecer, além de avaliação e orientação, reabilitação vocal (20042005), correlacionando os dois grupos acerca de seus hábitos, sintomas vocais e condições de trabalho.

\section{MÉTODOS}

O estudo apresentado é observacional transversal prospectivo. Para a sua realização foram analisadas 411 anamneses fonoaudiológicas aplicadas no momento da avaliação vocal de professores que procuraram o Programa de Saúde Vocal do SINPRO-SP entre março de 2001 e outubro de 2005, previamente à submissão dos avaliados a quaisquer das abordagens fonoaudiológicas (profilática ou terapêutica). Entre os anos de 2001 e 2003 o PSV estava sendo estruturado e oferecia aos professores avaliação vocal e orientações preventivas. Já de 2004 a 2005 este foi reestruturado e passou a oferecer, além de avaliação e orientações, reabilitação vocal. Assim, a divulgação do programa foi modificada, com o intuito de atrair para o programa tanto professores que desconheciam medidas vocais preventivas quanto aqueles com alteração vocal. Após dois anos da reestruturação do PSV, surgiu a necessidade de investigar se a oferta de uma abordagem diferencial favoreceria a procura do PSV por professores com perfil distinto daqueles que procuraram o PSV anteriormente. Para isso, os professores avaliados foram divididos em dois grupos:

Grupo 1 - composto por 256 profissionais que buscaram o serviço entre março de 2001 e dezembro de 2003, sendo 42 do sexo masculino e 214 do feminino, com media de idade de 46 anos.

Grupo 2 - composto de 155 profissionais que procuraram o serviço entre fevereiro de 2004 e outubro de 2005, sendo 29 do sexo masculino e 126 do feminino, com media de idade de 39,4 anos.

Como critério de inclusão, todos os professores precisavam estar exercendo a profissão.
A comparação entre os grupos foi feita baseada nos dados sobre as condições de trabalho, hábitos e sintomas vocais, os quais foram investigados durante as anamneses fonoaudiológicas aplicadas na avaliação vocal. Todas as anamneses foram aplicadas pela mesma fonoaudióloga e constaram de perguntas fechadas que diziam respeito a dados de identificação (sexo, faixa etária), organização e ambiente de trabalho (grau de ensino em que o professor lecionava, média de alunos por sala de aula, carga horária diária de trabalho e presença de ruído no ambiente de trabalho), procura por profissionais de saúde frente a alterações vocais (fonoaudiólogo ou otorrinolaringologista), hábitos que influenciam na saúde vocal (presença de cuidado com a voz, presença de uso vocal excessivo extraprofissional, tabagismo e freqüência no consumo de bebidas alcoólicas), identificação de sintomas vocais (presença de dor ou irritação na garganta, necessidade de pigarrear, dor ou tensão em região de coluna cervical, rouquidão devido ao uso vocal e variações da voz ao longo do dia).

As questões investigadas foram correlacionadas com o número de sintomas vocais (até 2, 3 e 4 ou mais) apresentado por cada professor.

A presente pesquisa foi aprovada pelo comitê de ética do Centro de Estudos da Voz - CEV (processo número 1412/04) e pelo SINPRO-SP.

Os cálculos das médias simples e porcentagens foram realizados por meio do programa computadorizado Microsoft Excel (Office 98). Para o estudo estatístico utilizou-se o programa computadorizado SPSS (Statistical Package for Social Sciences) - versão 13.0, aplicando-se o Teste de Mann-Whitney para a comparação entre grupos. O nível de significância adotado para a aplicação dos testes estatísticos deste estudo foi de $5 \%$ $(p=0,050)$.

\section{RESULTADOS}

Observando o número de sintomas em relação ao sexo (Tabela 1), a incidência de 4 ou mais sintomas vocais para o grupo 1 (G1) foi equivalente à 7\% (18) nos homens e 50\% (128) nas mulheres. Para o grupo 2 (G2) esta incidência foi de 17,40\% (27) nos homens e $80,65 \%$ (125) nas mulheres $(p=0,550)$.

Os professores que mais procuraram o PSV tinham idades entre 31 e 40 anos (Tabela1) - 42\% (108) do G1 e 37,42\% (58) do G2. Em segundo lugar observou-se 0 grupo daqueles entre $41 \mathrm{e}$ 50 anos - 26\% (67) do G1 e $25,16 \%$ (39) do G2, evidenciando uma população relativamente jovem para ambos os grupos $(p=0,557)$. 
Tabela 1 - Distribuição numérica e percentual dos sintomas de acordo com dados de identificação

\begin{tabular}{|c|c|c|c|c|c|c|c|c|c|c|c|c|c|c|c|c|}
\hline \multirow{4}{*}{ Dados de identificação } & \multicolumn{16}{|c|}{ Número de sintomas } \\
\hline & \multicolumn{4}{|c|}{$-/ 2$} & \multicolumn{4}{|c|}{3} & \multicolumn{4}{|c|}{$4 /-$} & \multicolumn{4}{|c|}{ Total } \\
\hline & \multicolumn{2}{|c|}{ Grupo 1} & \multicolumn{2}{|c|}{ Grupo 2} & \multicolumn{2}{|c|}{ Grupo 1} & \multicolumn{2}{|c|}{ Grupo 2} & \multicolumn{2}{|c|}{ Grupo 1} & \multicolumn{2}{|c|}{ Grupo 2} & \multicolumn{2}{|c|}{ Grupo 1} & \multicolumn{2}{|c|}{ Grupo 2} \\
\hline & $\mathbf{N}$ & $\%$ & $\mathbf{N}$ & $\%$ & $\mathbf{N}$ & $\%$ & $\mathbf{N}$ & $\%$ & $\mathbf{N}$ & $\%$ & $\mathbf{N}$ & $\%$ & $\mathbf{N}$ & $\%$ & $\mathbf{N}$ & $\%$ \\
\hline \multicolumn{17}{|l|}{ Sexo } \\
\hline Masculino & 11 & 4 & 2 & 1,3 & 13 & 5 & 0 & 0 & 18 & 7 & 27 & 17,4 & 42 & 16 & 29 & 18,7 \\
\hline Feminino & 45 & 18 & 0 & 0 & 41 & 16 & 1 & 0,65 & 128 & 50 & 125 & 80,7 & 214 & 84 & 126 & 81,3 \\
\hline \multicolumn{17}{|l|}{ Faixa etária } \\
\hline 20 a 30 & 6 & 2 & 0 & 0 & 8 & 3 & 0 & 0 & 33 & 13 & 35 & 22,6 & 47 & 18 & 35 & 22,6 \\
\hline 31 a 40 & 22 & 9 & 2 & 1,35 & 24 & 9 & 0 & 0 & 62 & 24 & 56 & 36,1 & 108 & 42 & 58 & 37,4 \\
\hline 41 a 50 & 17 & 7 & 0 & 0 & 15 & 6 & 0 & 0 & 35 & 14 & 39 & 25,2 & 67 & 26 & 39 & 25,2 \\
\hline 51 a 60 & 10 & 4 & 0 & 0 & 6 & 2 & 0 & 0 & 12 & 5 & 16 & 10,3 & 28 & 11 & 16 & 10,3 \\
\hline acima de 60 & 1 & 0 & 0 & 0 & 1 & 0 & 1 & 0,65 & 4 & 2 & 6 & 3,87 & 6 & 2 & 7 & 4,52 \\
\hline
\end{tabular}

Teste de Mann-Whitney:

Sexo $-p=0,550$; Faixa etária $-p=0,557$

A maior parcela dos professores $-\mathrm{G} 1=20 \%$ (51), G2 = 37,42\% (58) -dedicava-se a mais de uma série de ensino e apresentava ou mais sintomas vocais $(p=0,345)$. No $G 1$, a segunda maior parcela de professores, também com 4 ou mais sintomas vocais, lecionava para ensino fundamental I (10\%, 26), enquanto que, para o G2, lecionava para ensino superior $(17,40 \%, 27)$.

Independente do grau de ensino e número de sintomas houve predominância de até 30 alunos por sala de aula em ambos os grupos de professores: $56 \%$ (143) do G1 e 56,77\% (88) do G2 $(p=0,521)-$ Tabela 2 .
A maior parte do G1 (55\% - 141) lecionava por 1 período, enquanto a maioria do G2 $(47,74 \%-74)$ trabalhava 2 períodos por dia $(p<0,001)$. Observase também que 1\% (2) do G1 lecionava por 3 períodos diários (mais de 10 horas), em contrapartida ao G2, em que $10,97 \%$ (17) de indivíduos trabaIhava 3 períodos (Tabela 2). Quando analisados por número de sintomas, a maior parcela de ambos os grupos - G1=29\% (73) e G2=47,74\% (74) queixouse de 4 ou mais sintomas vocais e lecionavam por 2 períodos diários (Tabela 2).

Ambos os grupos relataram a presença de ruído no ambiente de trabalho: $\mathrm{G} 1=76 \%$ (195) e G2 = $78,07 \%(121) . \quad(p=0,660)-$ Tabela 2.

Tabela 2 - Distribuição numérica e percentual dos sintomas de acordo com características profissionais

\begin{tabular}{|c|c|c|c|c|c|c|c|c|c|c|c|c|c|c|c|c|}
\hline \multirow{4}{*}{ Aspectos profissionais } & \multicolumn{16}{|c|}{ Número de sintomas } \\
\hline & \multicolumn{4}{|c|}{$-/ 2$} & \multicolumn{4}{|c|}{3} & \multicolumn{4}{|c|}{$4 /-$} & \multicolumn{4}{|c|}{ Total } \\
\hline & \multicolumn{2}{|c|}{ Grupo 1} & \multicolumn{2}{|c|}{ Grupo 2} & \multicolumn{2}{|c|}{ Grupo 1} & \multicolumn{2}{|c|}{ Grupo 2} & \multicolumn{2}{|c|}{ Grupo 1} & \multicolumn{2}{|c|}{ Grupo 2} & \multicolumn{2}{|c|}{ Grupo 1} & \multicolumn{2}{|c|}{ Grupo 2} \\
\hline & $\mathbf{N}$ & $\%$ & $\mathbf{N}$ & $\%$ & $\mathbf{N}$ & $\%$ & $\mathrm{~N}$ & $\%$ & $\mathbf{N}$ & $\%$ & $\mathbf{N}$ & $\%$ & $\mathbf{N}$ & $\%$ & $\mathbf{N}$ & $\%$ \\
\hline \multicolumn{17}{|l|}{ Grau de ensino } \\
\hline Ensino infantil & 10 & 4 & 0 & 0 & 9 & 4 & 0 & 0 & 20 & 8 & 16 & 10,3 & 39 & 15 & 16 & 10,3 \\
\hline Ensino fundamental I & 14 & 5 & 0 & 0 & 13 & 5 & 0 & 0 & 26 & 10 & 27 & 17,4 & 53 & 21 & 27 & 17,4 \\
\hline Ensino fundamental II & 3 & 1 & 1 & 0,65 & 4 & 2 & 0 & 0 & 22 & 9 & 19 & 12,3 & 29 & 11 & 20 & 12,9 \\
\hline Ensino médio & 4 & 2 & 0 & 0 & 5 & 2 & 0 & 0 & 11 & 4 & 4 & 2,6 & 20 & 8 & 4 & 2,6 \\
\hline Ensino superior & 8 & 3 & 1 & 0,65 & 7 & 3 & 1 & 0,65 & 11 & 4 & 27 & 17,4 & 26 & 10 & 29 & 18,7 \\
\hline Cursos livres & 2 & 1 & 0 & 0 & 2 & 1 & 0 & 0 & 5 & 2 & 1 & 0,65 & 9 & 4 & 1 & 0,65 \\
\hline Atuação em 2 ou mais graus & 15 & 6 & 0 & 0 & 14 & 5 & 0 & 0 & 51 & 20 & 58 & 37,4 & 80 & 31 & 58 & 37,4 \\
\hline \multicolumn{17}{|l|}{ Número de alunos/sala aula } \\
\hline até 30 & 34 & 13 & 1 & 0,65 & 35 & 14 & 0 & 0 & 74 & 29 & 87 & 56,1 & 143 & 56 & 88 & 56,8 \\
\hline de 31 a 60 & 17 & 7 & 1 & 0,65 & 13 & 5 & 1 & 0,65 & 62 & 24 & 57 & 36,8 & 92 & 36 & 59 & 38,1 \\
\hline 61 ou mais & 5 & 2 & 0 & 0 & 6 & 2 & 0 & 0 & 10 & 4 & 8 & 5,16 & 21 & 8 & 8 & 5,16 \\
\hline \multicolumn{17}{|l|}{ Carga horária diária de trabalho } \\
\hline 1 período (até 5 horas) & 37 & 14 & 1 & 0,65 & 33 & 13 & 0 & 0 & 71 & 28 & 63 & 40,6 & 141 & 55 & 64 & 41,3 \\
\hline $\begin{array}{l}2 \text { períodos ( } 6 \text { a } 10 \text { horas) } \\
3 \text { períodos (superior a } 10\end{array}$ & 19 & 7 & 0 & 0 & 21 & 8 & 0 & 0 & 73 & 29 & 74 & 47,7 & 113 & 44 & 74 & 47,7 \\
\hline horas) & 0 & 0 & 1 & 0,65 & 0 & 0 & 1 & 0,65 & 2 & 1 & 15 & 9,67 & 2 & 1 & 17 & 11 \\
\hline \multicolumn{17}{|l|}{ Ruído em ambiente de trabalho } \\
\hline $\begin{array}{l}\text { Presente } \\
\text { Pro }\end{array}$ & 40 & 16 & 2 & 1,3 & 36 & 14 & 1 & 0,65 & 119 & 46 & 118 & 76,1 & 195 & 76 & 121 & 78,1 \\
\hline Ausente & 16 & 6 & 0 & 0 & 18 & 7 & $\square \square$ & 0 & 27 & 11 & 34 & 21,9 & 61 & 24 & 34 & 21,9 \\
\hline
\end{tabular}

Teste de Mann-Whitney:

Grau de ensino $-p=0,345$; Número de alunos/sala aula $-p=0,521$; Carga horária de trabalho $-p<0,001$; Ruído em ambiente de trabalho $-p=0,660$ 
De acordo com a Tabela 3, observa-se que $49 \%$ (125) do G1 e $68,38 \%$ (106) do G2 procuraram assistência otorrinolaringológica e/ou fonoaudiológica devido a alterações vocais $(p<0,001)$. Mesmo com queixa de 4 ou mais sintomas vocais, $27 \%$ (69) do G1 e 30,32\% (47) do G2 não procuraram ajuda especializada.

Observa-se que $38 \%$ (97) do G1 e 43,87\% (68) do G2 negaram medidas de cuidados com a voz ( $p=0,231$ ). Porém, 36\% (92) do G1 e 54,83\% (85) do $\mathrm{G} 2$ relataram cuidados com a voz, mas apresentaram 4 ou mais sintomas vocais (Tabela 3 ).

Quanto à utilização vocal intensa em atividades extra-curriculares, $61 \%$ (155) do G1 e 58,71\% (91) do G2 relataram tal hábito, prevalecendo 4 ou mais sintomas vocais em ambas amostras $(p=0,713)$ Tabela 3.

Em relação ao tabagismo (Tabela 3), 15\% (38) do grupo G1 relataram fumar, enquanto uma parcela menor $-6,45 \%(10)$ - do G2 eram fumantes $(p=0,010)$.

Apesar da maior parte dos professores de ambos os grupos negarem o consumo de bebidas alcoólicas (Tabela 3) sua utilização regular foi maior no G1: 5\% (14) comparando-se aos 1,93\% (3) do $\mathrm{G} 2(p=0,029)$.

Tabela 3 - Distribuição numérica e percentual dos sintomas de acordo com hábitos investigados

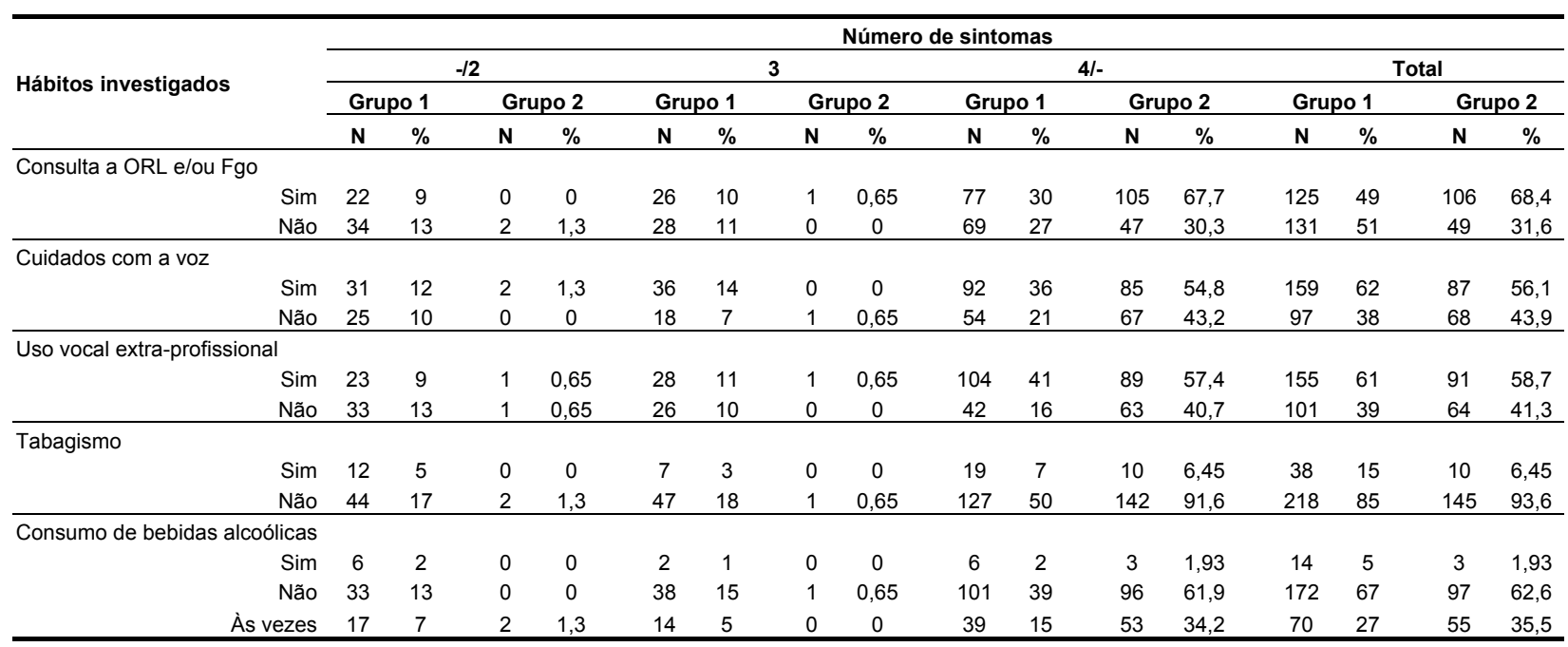

Teste de Mann-Whitney:

Consulta a ORL e/ou Fgo $-p<0,001$; Cuidados com a voz $-p=0,231$; Uso vocal extra-profissional $-p=0,713$; Tabagismo $p=0,010$; Consumo de bebidas alcoólicas $-p=0,029$

\section{DISCUSSÃO}

Há anos a voz do professor tem merecido atenção dos fonoaudiólogos, especialmente pela procura à assistência de especialistas frente a alterações vocais ${ }^{7}$. Orientações sobre saúde vocal geralmente não fazem parte da formação do professor, contribuindo para o despreparo ao enfrentar a demanda de voz na docência ${ }^{1,8}$. Tal fato favorece a presença de alterações vocais por desconhecimento de medidas preventivas ou de como agir aos primeiros sinais e sintomas de alterações vocais, perdurando assim o quadro de alteração e prorrogando o início do tratamento ${ }^{3,9}$.

É certo que os fatores de risco para o desenvolvimento de um transtorno vocal ocupacional compreendem tanto as características individuais quanto ambientais e emocionais, contemplando o indivíduo como um todo e o local onde ele está inserido 10-12. Assim, para compreender as características dos professores que procuraram o Programa de Saúde Vocal (PSV) do SINPRO-SP em dois momentos diferentes, comparou-se os perfis dos professores que recorreram ao PSV de 2001 à 2003 (fonoaudiologia preventiva - grupo 1) e 2004 à 2005 (fonoaudiologia preventiva e terapêutica - grupo 2), correlacionando-os de acordo com as condições de trabalho, hábitos e sintomas vocais.

A média de sintomas vocais para o grupo 1 (G1) foi de 3,5 e para o grupo 2 (G2) foi de 5,8. Se o número médio de sintomas vocais encontrados em pesquisas com professores foi de $4,3{ }^{13}$, observase então que o G2 apresentou um número elevado de sintomas, o que pode indicar que os professores 
desse grupo procuraram o programa já com uma alteração vocal. Independente de outras características, 57\% (146) do G1 e 98,05\% (152) do G2 apresentaram 4 ou mais sintomas vocais - tabela 1 ( $p<0,001)$. Este número elevado de sintomas vocais nos professores pode refletir uma procura tardia à assistência fonoaudiológica, por vezes com alteração vocal já instalada. Sabe-se que o número de sintomas não diferencia vozes neutras de alteradas, mas é preciso valorizar os sintomas vocais nos programas de prevenção voltados aos professores, para que estes indivíduos possam identificálos e prevenir possíveis alterações vocais ${ }^{14}$.

Houve predomínio de mulheres em ambos os grupos (Tabela 1), sendo que $50 \%$ (128) do G1 e $80,65 \%$ (125) do G2 relataram 4 ou mais sintomas vocais $(p=0,550)$. A população de professores em geral é predominantemente feminina ${ }^{6}$, justificando a grande representatividade deste sexo nas pesquisas. Ainda, os professores que mais procuraram o PSV em ambos grupos caracterizaram-se por adultos jovens (entre 31 e 40 anos de idade) - Tabela 1. Isso indica que, mesmo sendo jovem, o acúmulo das atividades profissionais pode favorecer um número elevado de sintomas vocais, possivelmente prejudicando a saúde vocal do professor.

Embora alguns estudos não tenham observado relação entre a frequência de disfonia e idade ou tempo de profissão do professor ${ }^{10}$, acredita-se que a idade influencie a presença de um quadro de disfonia ${ }^{15}$, já que a faixa etária entre 31 e 50 anos de idade condiz com o perfil de professores que lecionavam para mais de um nível de ensino (educação infantil e fundamental, fundamental I e II, fundamental e médio) ou para o ensino superior $^{1,19,5}$ - tabela 1. A maior parcela dos professores do $\mathrm{G} 1=20 \%$ (51) e do $\mathrm{G} 2=37,42 \%$ (58) se dedicava a mais de uma série de ensino e apresentava 4 ou mais sintomas vocais - tabela 2 $(p=0,345)$. Assim, ministrando aulas para diferentes níveis de ensino, o professor exerce diferentes demandas de voz, já que cada um dos graus exige recursos pedagógicos específicos (estratégias de aula) e uma postura diferenciada perante os alunos ${ }^{1 ;}$; favorecendo os sintomas vocais.

A reestruturação do serviço fonoaudiológico oferecido pelo sindicato favoreceu o aumento de sua divulgação por meio de visitas de funcionários do SINPRO-SP às universidades e informativos via boletins eletrônicos, o que provavelmente contribuiu para o aumento na porcentagem de professores do ensino superior no G2 - 18,70\% (29) freqüentando o PSV (Tabela 2). Considera-se que os professores universitários em geral falam por várias horas, competem com ruído ambiental, tendem a elevar a intensidade e freqüência de voz causando maior esforço à fonação e fadiga vocal ${ }^{16}$. Tais fatos podem justificar o número elevado de sintomas vocais apresentados pelos professores de ensino superior do G2, que geralmente não lecionavam para outros níveis de ensino. De fato, não é possível afirmar que os professores universitários apresentaram mais sintomas vocais em decorrência de alterações vocais ocupacionais, mas que possivelmente passaram a procurar mais o atendimento fonoaudiológico após obterem informações sobre o serviço oferecido pelo SINPROSP, fato que destaca a importância da divulgação da atuação fonoaudiológica com professores.

Os professores de educação infantil e ensino fundamental são os que mais se associam ao SINPRO-SP, o que nos faz questionar se estes professores consultaram o serviço por possuírem mais alterações ou por serem os que mais se sindicalizaram e, conseqüentemente, obtiveram mais acesso ao serviço ${ }^{4}$.

Independente do grau de ensino, houve predominância de até 30 alunos por sala de aula em ambos os grupos de professores $-p=0,521$ (Tabela 2). Considerando-se que a maior parte dos professores lecionava para mais de um nível de ensino e com salas de até 30 alunos, provavelmente sem a utilização de aparelho amplificação vocal individual (microfone) e em ambientes desfavoráveis física e acusticamente ${ }^{1}$, é justificável o número elevado de sintomas vocais apresentados por estes indivíduos; já que a voz precisa ser projetada para que todos os alunos a escutem e também deve superar o ruído ambiental. Além disso, observa-se uma tendência à redução da quantidade de salas de aulas ${ }^{4}$, resultando em diminuição no número de docentes por escola e aumento do número de alunos por sala de aula: 36\% (92) do G1 e 38,07\% (59) do G2 lecionavam para um número de alunos que variava de 30 a 60 (Tabela 2) - aumentando os desgastes psíquicos, físicos e vocais do professor.

Considerando-se a carga horária diária de trabalho dos professores de cada grupo, observouse que houve diferença entre estes, pois a maior parte do G1 - 55\% (141) - trabalhava 1 período e $44 \%$ (113) trabalhavam 2 períodos, sendo que no G2 a carga horária predominante foi de 2 períodos 47,74\% (74) - Tabela 2. Esta divergência de carga horária entre os dois grupos $(p<0,001)$ favorece a hipótese de que a atuação dos professores em mais de dois níveis de ensino faz com que tenham uma maior demanda vocal e consequentemente propicia o surgimento de sintomas vocais. Usar a voz por horas seguidas associando-se à tensão muscular e intensidade vocal elevada no ambiente escolar pode levar a modificações vocais em razão da fadiga muscular e atrito entre as pregas vocais, 
alterando o padrão de vibração da mucosa ${ }^{5} \mathrm{e}$ aumentando o número de sintomas vocais.

Quanto ao ambiente de trabalho, observa-se na Tabela 2 presença de ruído ambiental para ambos os grupos $(p=0,660)$. Tais dados refletem parte das condições de trabalho do professor e a dificuldade em modificá-las, o que significa que as manifestações vocais não dependem exclusivamente de questões comportamentais, e sim de um conjunto de fatores associados: uso vocal, condições de trabalho, estado emocional e conhecimento acerca da saúde vocal ${ }^{17}$. Portanto, não é possível afirmar que o ruído foi exclusivamente o fator causal dos sintomas vocais, porém deve-se considerar que interfere mantendo ou aumentando o desgaste vocal do professor ${ }^{18}$. O elevado índice de queixas quanto ao ruído ambiental é um fator preocupante, visto que pode levar à situação de competição sonora já que há necessidade de aumentar a intensidade de voz a fim de superar o efeito de mascaramento sonoro. Este ato favorece a fadiga vocal frente à tentativa de manter a devida inteligibilidade do discurso por vezes associando-se à técnica vocal inadequada ${ }^{5,19}$. Assim, as condições do ambiente de trabalho dos professores têm um papel importante na presença de sinais e sintomas vocais.

Em diversos países os professores ocupam a categoria mais suscetível a procurar um otorrinolaringologista acerca de problemas vocais ${ }^{13,20}$, muitas vezes quando já apresentam algum comprometimento de voz ${ }^{4}$. Observou-se na amostra de professores analisados que a porcentagem em relação à procura por especialista aumentou: de 49\% (125) - G1 - passou para 68,38\% (106) G2, como demonstrado na Tabela 3 ( $p<0,001)$. Provavelmente este aumento pela procura do serviço fonoaudiológico oferecido pelo SINPRO-SP ocorreu em decorrência da reestruturação do PSV da instituição, que passou a oferecer também tratamento. Considerando que alguns professores não possuem conscientização sobre aspectos relacionados à saúde vocal, pode-se esperar que desconheçam e não pratiquem medidas de cuidados com a própria voz (Tabela 3): 38\% (97) dos indivíduos do G1 e $43,87 \%$ (68) do G2 negaram cuidados com a voz $(p=0,231)$. Alguns professores tomam medidas para cuidar da voz quando já sentem impacto em seu rendimento vocal ao lecionar. Observou-se que $36 \%$ (92) do G1 e $54,83 \%$ (85) do G2 relataram cuidados com a voz, mas apresentaram quatro ou mais sintomas vocais, evidenciando que apenas 0 cuidado com a voz não garante a saúde vocal, já que nem sempre as medidas tomadas são efetivas à saúde da voz (Tabela 3 ).
A maior parte de ambos os grupos (Tabela 3) relatou o uso vocal extra-profissional. Neste caso, o uso vocal excessivo fora do ambiente de trabalho diminui o tempo de repouso vocal e pode ser mais um dos fatores mantenedores dos sintomas vocais apresentados pelos professores. Comparando-se os dois grupos (Tabela 3), houve uma diminuição na porcentagem dos indivíduos que usavam a voz excessivamente, mas de fato prevaleceu um elevado número de sintomas, com 4 ou mais em ambas amostras $(p=0,713)$. Sabe-se que a fala excessiva constitui um dos fatores que mais causam alterações na $\operatorname{voz}^{21}$ por exigir ação prolongada do aparelho fonatório, levando a possível fadiga muscular e vocal em decorrência da diminuição de repouso de voz ${ }^{7}$.

Em relação ao tabagismo, observou-se uma parcela pequena de professores fumantes para ambos os grupos (Tabela 3). De acordo com estudos epidemiológicos no Brasil ${ }^{22}$, os indivíduos do sexo masculino apresentam maior prevalência de tabagismo do que os do feminino, sendo que as causas para tal fato são atribuídas a fatores históricos e culturais. Desde que o tabagismo foi introduzido na sociedade moderna, a proporção de homens fumantes tem sido mais elevada do que a de mulheres. Portanto, como a amostra dos grupos foi composta principalmente por mulheres, provavelmente justifica-se o número reduzido de indivíduos fumantes. Ainda assim, não é possível afirmar que o tabagismo está diretamente relacionado ao surgimento de sintomas vocais na amostra pesquiada, mas vale ressaltar que tal hábito constitui um dos fatores agressivos para o organismo em geral e para a laringe, prejudicando a voz.

Apesar da maior parte dos professores de ambos os grupos negarem o consumo de bebidas alcoólicas, sua utilização regular foi mais significativa para o G1 do que para o G2 (Tabela 3). Diante disso, aponta-se a importância de que esses profissionais sejam informados sobre os hábitos nocivos para a voz ${ }^{6}$.

Os professores possuem necessidades específicas quanto à assistência fonoaudiológica, tanto em relação aos aspectos comportamentais quanto aos relacionados ao ambiente de trabalho ${ }^{22,23}$. Desta forma, é preciso considerar a promoção da saúde vocal desta categoria profissional nos serviços voltados à saúde do trabalhador ${ }^{24-27}$. É importante também que os professores recebam informações primárias em sua formação, com disciplinas e/ou palestras sobre voz ${ }^{28-30}$. Tais informações proporcionariam maiores chances do futuro profissional da voz reconhecer e evitar alterações vocais e conhecer quais medidas tomar frente a sinais $\mathrm{e}$ sintomas de alterações de voz. 
Diante de todos os aspectos correlacionados e discutidos neste estudo pode-se conferir que os professores apresentaram um número significante de sintomas vocais e, em sua maioria, provavelmente procuraram assistência especializada quando existia uma alteração vocal iminente ${ }^{4,20}$. Fica claro que o PSV oferecido pelo SINPRO-SP deve manter a sua atuação preventiva e terapêutica, favorecendo assim a conscientização e (re) habilitação dos professores sindicalizados, contribuindo para a saúde vocal destes profissionais.

\section{CONCLUSÃO}

Comparando-se os grupos 1 e 2 de professores avaliados no Programa de Saúde Vocal do SINPRO-SP, conclui-se que o perfil do professor que recorreu ao atendimento na instituição diferenciou-se de acordo com a ampliação do foco de intervenção fonoaudiológica.

Os grupos assemelharam-se em relação ao sexo (feminino), faixa etária (entre 31 e 40 anos), lecionar para mais de um grau de ensino, com até 30 alunos por sala de aula, presença de ruído em ambiente de trabalho, praticar cuidados com a voz, uso vocal excessivo extra-profissional, não tabagismo e não consumo de bebidas alcoólicas frequentemente.

Os grupos divergiram quanto à:

- carga horária diária de trabalho: a maioria do G1 trabalhava até 5 horas, enquanto a do G2 trabaIhava de 6 à 10 horas/dia;

- busca por otorrinolaringologista e/ou fonoaudiólogo por alterações vocais: a maior parte do G1 não havia procurado um especialista, enquanto a do G2 já havia procurado assistência profissional.

Observou-se também uma média maior de sintomas no G2 quando comparado ao G1, o que mostra que esse grupo procurou o PSV com um maior risco de alteração vocal. É provável que o G2 possuísse mais professores que necessitavam de reabilitação vocal por sofrerem acúmulo de atividades (principalmente trabalhar mais horas por dia) e desconhecerem medidas preventivas.

Assim, é muito importante que existam programas de saúde vocal para o professor com o foco na prevenção e informação. Porém, deve-se considerar que muitos professores já apresentam sintomas e alterações vocais e necessitam de acesso ao tratamento, o qual contribuirá tanto para a qualidade de trabalho quanto de vida desses profissionais.

\begin{abstract}
Purpose: to analyze vocal symptoms from two groups of teachers assessed during two different instances of a vocal health program. Methods: correlate work conditions and habits with the number of vocal symptoms submitted by 411 teachers divided into G1 (256 subjects to be submitted to a prevention program) and G2 (155 subjects to be submitted to a prevention and treatment program). Results: it was observed that in both groups there was a larger number of women $(p=0.550)$, aged 31 to 40 years $(p=0.557)$, teaching for more than one grade $(p=0.345)$ and with up to 30 students per class $(p=0.521)$; they related presence of noise in their work environment $(p=0.660)$, used to take care of their voices $(p=0.231)$, were non-smokers $(p=0.010)$, used their voices in extra-professional activities and did not have the habit of drinking. On the other hand, both groups were different upon relating daily work hours; in G1 most teachers worked up to 5 hours a day, and in G2 most of them used to work 6 to 10 hours a day. G1 had $51 \%$ of individuals who did not search for a laryngologist's or speech pathologist's help when needed while in G2 a higher percentage of individuals (68.38\%) had already looked for a specialist due to voice disorders. Both groups had a large number of voice symptoms ( $\geq$ 4), in $\mathrm{G} 1$ the mean number of symptoms was 3.5 while in $\mathrm{G} 2$ it was 5.8 ; demonstrating a statistically higher percentage of symptoms in G2 (98.05\%) than in G1 (57\%) - p<0.001. Conclusion: although both groups had similar profiles, a higher mean of vocal symptoms was found in G2, meaning that this group looked for the speech pathology assistance already with a higher risk of voice disorders, possibly due to the usage of a different type of intervention (by offering a vocal rehabilitation), which attracted teachers with more disorders. Thus, it is very important to offer vocal health programs focusing both prevention as well as vocal treatment, because these will contribute not only to the subjects' work but also to their quality of life.
\end{abstract}

KEYWORDS: Voice; Symptoms; Habits; Working Conditions; Voice Disorders 


\section{REFERÊNCIAS}

1. Servilha EAM. Consciência vocal em docentes universitários. Pró-Fono. 1997; 9(2):53-61.

2. Fuess VLR, Lorenz MC. Disfonia em professores do ensino municipal: prevalência e fatores de risco. Rev Bras Otorrinolaringol. 2003; 69(6):807-12.

3. Schwarz K, Cielo CA. A voz e as condições de trabalho de professores de cidades pequenas do Rio Grande do Sul. Rev Soc Bras Fonoaudiol. 2005; 10(2):83-90.

4. Zambon FC. Sintomas vocais, hábitos e condições de trabalho dos professores atendidos no SINPRO-SP. [monografia] São Paulo (SP): Centro de Estudos da Voz; 2005.

5. Pordeus AMJ, Palmeira CT, Pinto VCV. Inquérito de prevalência de problemas da voz em professores da universidade de fortaleza. Pró-Fono. 1996; 8(2):15-24.

6. Grillo MHMM, Penteado RZ. Impacto da voz na qualidade de vida de professore(a)s do ensino fundamental. Pró-Fono. 2005; 17(3): 321-330.

7. Dragone ML, Behlau M. A fonoaudiologia brasileira e a voz do professor: olhares científicos no decorrer do tempo. Rev Fonoaudiol Bras. 2006; 4(2):6-8.

8. Behlau M. Voz: o livro do especialista. Vol. 2. Rio de Janeiro: Revinter; 2005.

9. Roy N, Merrill RM, Thibeault S, Gray SD, Smith EM. Voice disorders in teachers and the general population: effects on work performance, attendance, and future career choices. J Speech Lang Hear Res. 2004; 47(3):542-51.

10. Sliwinska-Kowalska M, Niebudek-Bogusz E, Fiszer M, Los-Spychalska T, Kotylo P, SznurowskaPrzygocka B, Modrzewska M. The prevalence and risk factors for occupational voice disorders in teachers. Folia Phoniatr Logop. 2006; 58(2):85-101. 11. Delcor NS, Araújo TM, Reis EJFB, Porto LA, Carvalho FM, Silva MO, Barbalho L, Andrade JM. Labor and health conditions of private school teachers in Vito'ria da Conquista, Bahia, Brazil. Cad Sau'de Pu'blica. 2004; 20(1):187-196.

12. Zambon FC, Behlau M, Roy N. Considerações preliminares sobre um levantamento epidemiológico brasileiro de distúrbios vocais em professores. In: XI Simpósio Internacional do Centro de Estudos da Voz (CEV). A Voz do Professor. Evidências Científicas da Intervenção Fonoaudiológica. Material impresso. São Paulo / SP; 2006. p. 31.

13. Nagano L, Dragone MLS, Behlau M. Estudo sobre a relação entre qualidade vocal e sintomas vocais. In: Intercâmbio de pesquisas em linguística aplicada. Caderno de Resumos. São Paulo; 1998.
14. Tavares ELM, Martins RHG. Vocal Evaluation in Teachers With or Without Symptoms. J Voice. 2006; 21(4):407-414.

15. Roy N, Merrill RM, Thibeault S, Parsa R, Gray SD, Smith EM. Prevalence of voice disorders in teachers and the general population. J Speech Lang Hear Res. 2004; 47(2):281-93.

16. Giannini SPP, Passos MC. Histórias que fazem sentidos: as determinações das alterações vocais do professor. Dist Comun. 2006; 18(2):245-57.

17. Medeiros AM, Barreto SM, Assunção AA. Voice disorders (dysphonia) in public school female teachers working in Belo Horizonte: prevalence and associated factors. J Voice. 2007; 22(6):676-87.

18. Verdoline K, Ramig LO. Review: occupacional risks for voice problems. Logoped Phoniatr Vocol. 2001; 26(1):37-46.

19. Brasil. Ministério da Saúde (2004). Secretaria de Vigilância em Saúde. Secretaria de Atenção à Saúde. Instituto Nacional de Câncer. Coordenação de Prevenção e Vigilância. Inquérito domiciliar sobre comportamentos de risco e morbidade referida de doenças e agravos não transmissíveis: Brasil, 15 capitais e Distrito Federal, 2002-2003 Rio de Janeiro: INCA. [acessado em 31 julho 2006]. Disponível em: URL: http://www.inca.gov.br/ inquerito/docs/tab.pdf.

20. de Jong FI, Kooijman PG, Thomas G, Huinck WJ, Graamans K, Schutte HK. Epidemiology of voice problems in Dutch teachers. Folia Phoniatr Logop. 2006; 58(3):186-98.

21. Ferreira L, Latorre M, Giannini S, Ghirardi A, Karmann D, Silva E, Figueira S ; Ferreira L. Influence of Abusive Vocal Habits, Hydration, Mastication, and Sleep in the Occurrence of Vocal Symptoms in Teachers. J Voice. 2009/ahead of print.

22. Jardim R, Barreto SM, Assunção AA. Condições de trabalho, qualidade de vida e disfonia entre docentes.Cad Saúde Pública. 2007; 23(10): 2439-2461.

23. Penteado RZ, Rossi D. Vivência de voz e percepções de professores sobre saúde vocal e trabalho. Saúde em Revista. 2006; 8(18): 39-48.

24. Gillivan-Murphy P, Drinnan MJ., O'Dwyer TP, Ridha $\mathrm{H}$, Carding P. The Effectiveness of a Voice Treatment Approach for Teachers With SelfReported Voice Problems. Journal of Voice. 2006; 20(3):423-431.

25. Kooijman PGC, Thomas G, Graamans K, de Jong FICRS. Psychosocial Impact of the Teacher's Voice Throughout the Career. J Voice. 2007; 21(3):316-324.

26. Silverio KCA, Gonçalves CGO, Penteado RZ, Vieira TPG, Libardi A, Rossi D. Ações em saúde vocal: proposta de melhoria do perfil vocal de 
professores. Pró-Fono Revista de Atualização Científica. 2008; 20(3):177-82.

27. Bovo R, Galceran M, Petruccelli J, Hatzopoulos S. Vocal Problems Among Teachers: Evaluation of a Preventive Voice Program. J Voice. 2007; 21(6):705-722.

28. Smolander S, Huttunen K. Voice problems experienced by Finnish comprehensive school teachers and realization of occupational health care. Logoped Phoniatr Vocol. 2006; 31(4):166-71.
29. Wingate JM, Brown WS, Shrivastav R, Davenport P, Sapienza CM. Treatment outcomes for professional voice users. J Voice. 2007;21(4):433-49.

30. de Medeiros AM, Barreto SM, Assunção AA. Voice disorders (dysphonia) in Public School Female Teachers Working in Belo Horizonte: prevalence and associated factors. J Voice. 2007/ article in press.

DOI: 10.1590/S1516-18462010005000075

RECEBIDO EM: 09/09/2009

ACEITO EM: 05/03/2010

Endereço para correspondência:

Karin Choi-Cardim

Rua Rudi Schaly, 126

São Paulo - SP

CEP: 05101-060

E-mail: karin_fga@ @otmail.com kathelly@ kbonet.com.br 